\title{
An investigation of the mechanism of dexmedetomidine in improving postoperative cognitive dysfunction from the perspectives of alleviating neuronal mitochondrial membrane oxidative stress and electrophysiological dysfunction
}

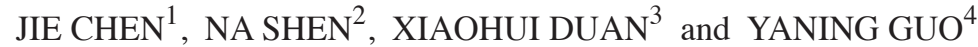 \\ ${ }^{1}$ Department of Anesthesiology, Affiliated Hospital of Hebei University of Engineering, Handan, Hebei 056029; \\ ${ }^{2}$ Department of ENT, Tianjin 4th Center Hospital, Tianjin 300140; ${ }^{3}$ Department of ENT, Affiliated Hospital \\ of Hebei University of Engineering, Handan, Hebei 056029; ${ }^{4}$ Department of Anesthesiology, \\ Handan Central Hospital, Handan, Hebei 056002, P.R. China
}

Received August 18, 2017; Accepted November 22, 2017

DOI: $10.3892 /$ etm.2017.5589

\begin{abstract}
The aim of this study was to investigate the mechanism of dexmedetomidine in improving postoperative cognitive dysfunction from the perspectives of alleviating neuronal mitochondrial membrane oxidative stress and electrophysiological dysfunction. A total of 120 patients undergoing elective surgery under general anesthesia from June 2013 to May, 2016 were selected as the subjects of the study and randomly divided into the propofol + remifentanil and dexmedetomidine groups. The Rey Auditory Verbal Learning Test (AVLT) and Beck Depression Inventory (BDI) were performed at day 1 before operation and at day 1, 3, 5 and 15 after operation. The mitochondrial membrane potential was detected using a flow cytometer after staining and labeling for mitochondria in leukocytes via JC-1 fluorescence staining using a fluorescence probe at day 1 before operation and at day 1,3,5 and 15 after operation. The activities of mitochondrial respiratory chain complexes at day 1 before and after operation were detected via enzyme-linked immunosorbent assay (ELISA). The results showed that there were no statistically significant differences in the comparisons of general conditions (age, body weight, sex ratio, body mass index, anesthesia time, operation time, and length of stay in the ICU and hospital) for the dexmedetomidine and propofol + remifentanil groups $(\mathrm{P}>0.05)$. At day 3 and 5 after operation, the National Institutes of Health Stroke Scale (NIHSS) scores and AVLT scores in the two groups were decreased in different degrees, but the decrease range in the dexmedetomidine group was smaller than that in the
\end{abstract}

Correspondence to: Dr Yaning Guo, Department of Anesthesiology, Handan Central Hospital, 15 Zhonghuanan Street, Handan, Hebei 056002, P.R. China

E-mail: yaning_guo123@163.com

Key words: dexmedetomidine, oxidative stress, electrophysiology, neurons, mitochondria, cognitive dysfunction propofol + remifentanil group, and the differences were statistically significant $(\mathrm{P}<0.05)$. At day 3,5 and 15 after operation, the BDI scores of the two groups were increased in different degrees, but the increase range in the dexmedetomidine group was smaller than that in the propofol + remifentanil group, and the differences were statistically significant $(\mathrm{P}<0.05)$. At day 1, 3 and 5 after operation, the mitochondrial membrane potentials of the two groups were decreased in different degrees, but the decrease range in the dexmedetomidine group was smaller than that in the propofol + remifentanil group, and the differences were statistically significant $(\mathrm{P}<0.05)$. The mitochondrial membrane potentials of the two groups returned to the preoperative levels at day 15 after operation. The activities of mitochondrial respiratory chain complex I-IV in the propofol + remifentanil group at day 1 after operation were significantly decreased compared with those before operation, and the differences were statistically significant $(\mathrm{P}<0.05)$. The decrease in activities of mitochondrial respiratory chain complex I-IV in the propofol + remifentanil group at day 1 after operation was more significant than that in the dexmedetomidine group, and the difference was statistically significant $(\mathrm{P}<0.05)$. The results suggest that dexmedetomidine can relieve neuronal damage that may be caused by mitochondrial membrane oxidative stress, alleviate the damage to mitochondrial related enzyme system activity, and reduce the damage to the activities of mitochondrial respiratory chain enzyme complex I, II, III and IV, ultimately improving the postoperative cognitive dysfunction of patients.

\section{Introduction}

The elderly may suffer from central nervous system complications after surgery, which are manifested as mental disorders, anxiety, personality changes, and memory and concentration decline. The postoperative changes in personality, social competence, cognitive ability and skills are known as postoperative cognitive dysfunction (POCD) (1). Studies have shown that the elderly with POCD after general anesthesia are dominated in 
patients in this age group (2). Under general anesthesia, the cerebral blood flow is significantly reduced, which disturbs the brain tissue metabolism and increases the permeability of blood-brain barrier, resulting in neuronal and brain glial cell damage, and eventually leading to POCD (3). The brain is the organ with the most oxygen consumption in the human body, and there are fewer antagonists against oxidative stress (such as catalase, glutathione and vitamin E) in the neurons of the central nervous system, thus, the neurons are susceptible to the attack of free radicals. The hippocampal neurons are rich in unsaturated fatty acids, so these neurons are particularly sensitive to oxidative stress $(4,5)$. In addition, oxidative stress can lead to a decline in mitochondrial membrane potential, ultimately resulting in neuronal apoptosis and even death (4). Maintaining mitochondrial balance via mitochondria, as the core of neuronal energy metabolism, is a prerequisite to maintaining the health of neurons (5). Studies have found that there is mitochondrial transport between neuronal cell bodies and axons; however, recent findings have shown that there is special mitochondrial transport between neuronal axons and glial cells, and astrocytes can swallow and degrade the mitochondria released by axons $(6,7)$. Numerous mitochondria are required to provide energy in the axonal transport process. Thus, the collapse of mitochondrial membrane potential may also lead to neuronal apoptosis directly (6). Dexmedetomidine can reduce the cerebral blood flow by reducing cerebral perfusion pressure, and does not affect the cerebral oxygen supply under low blood oxygen saturation, even in the process of overventilation. In addition, it can act as a neuroprotective agent for local cerebral ischemia or tissue hypoxia. When used as an anesthesia adjuvant, dexmedetomidine can improve the hemodynamic stability, and reduce the doses of anesthetics and analgesics during operation, thus playing a role in the prevention of POCD.

The present study aimed to investigate the mechanism of dexmedetomidine in improving POCD from the perspectives of alleviating neuronal mitochondrial membrane oxidative stress and electrophysiological dysfunction.

\section{Materials and methods}

General materials. A total of 120 patients undergoing elective surgery under general anesthesia in the Affiliated Hospital of Hebei University of Engineering from June, 2013 to May, 2016 were selected as the subjects of study and randomly divided into two groups using a random number table: propofol + remifentanil group $(n=60)$ and dexmedetomidine group $(n=60)$.

Inclusion criteria of patients. Inclusion criteria for patients undergoing elective surgery under general anesthesia were: i) Patients in either sex; ii) patients aged 40-70 years; iii) patients without a history of operation under general anesthesia; and iv) patients who signed the informed consent before surgery. This study was approved by the Ethics Committee of the Affiliated Hospital of Hebei University of Engineering (Hebei, China).

Exclusion criteria of patients. Exclusion criteria for the study were: i) Patients with a medical history of mitochondrial diseases; ii) patients with diseases of mental or nervous systems before surgery; iii) patients with severe vertebral artery or carotid artery stenosis; iv) patients with chronic obstructive pulmonary disease; v) patients with severe endocrinal and metabolic diseases; vi) patients with chronic heart, hepatic or renal failure; vii) patients with a mini-mental state examination score of $<24$ points before surgery; viii) patients with a history of drug/poison dependence; ix) patients with a medical history of epileptic seizure or limb convulsion, and $\mathrm{x}$ ) patients with a medical history of intracranial tumor, bleeding or craniocerebral trauma.

Treatment process. Under intravenous general anesthesia viatrachea cannula, all the patients were intramuscularly injected with $0.01 \mathrm{mg} / \mathrm{kg}$ midazolam (approval no. NMPN H10980025; Jiangsu Enhua Pharmaceutical Co., Ltd., Xuzhou, China) and $0.1 \mathrm{mg} / \mathrm{kg}$ morphine (approval no. NMPN H20013351; Northeast Pharm, Shenyang First Pharmaceutical Co., Ltd., Shenyang, China).

Patients lay on the operating table, and the veins were opened, followed by ECG monitoring, blood oxygen saturation monitoring, end-tidal carbon dioxide partial pressure and Bispectral index (BIS) detection (BIS VISTA Monitoring System ${ }^{\mathrm{TM}}$, Aspect Medical Systems, Norwood, MA, USA) and the arterial blood pressure was monitored via radial arterial catheterization. Patients in both groups were treated with midazolam (0.05-0.1 mg/kg), remifentanil (0.5-1.0 $\mu \mathrm{g} / \mathrm{kg})$, propofol $(1-2 \mathrm{mg} / \mathrm{kg})$ and etomidate $(0.1-0.5 \mathrm{mg} / \mathrm{kg})$ for fast intravenous anesthesia induction, and received oral endotracheal intubation after muscle relaxation was satisfactory. All the patients were treated with intermittent positive pressure mechanical ventilation with the inhaled oxygen concentration of 35-60\%, tidal volume of 5-7 ml/kg, respiratory rate of 9-12 times/min and peak airway pressure $<30 \mathrm{cmH}_{2} \mathrm{O}$. The blood gas monitoring was repeated, and the arterial partial pressure of oxygen was controlled within $150 \mathrm{mmHg}$, while carbon dioxide partial pressure was controlled at approximately $40 \mathrm{mmHg}$. Under the general anesthesia throughout the operation, remifentanil $(0.5-1 \mu \mathrm{g} / \mathrm{kg} / \mathrm{h})$ and propofol (3-10 $\mathrm{mg} / \mathrm{kg} / \mathrm{h})$ were intravenously administered to both groups. Dexmedetomidine $(0.2 \mu \mathrm{g} / \mathrm{kg} / \mathrm{h})$ was added to the dexmedetomidine group during induction and maintenance periods. The intravenous drug dose during operation was induced with the lowest concentration, and the current target concentration and the final target concentration of drugs in each group was adjusted according to the BIS maintained at 45-55 and the patient's hemodynamic state. Finally, the corresponding vasoactive drugs were applied according to cardiac function.

Observational indexes. The neurologic impairment of patients in the two groups at day 1 before operation and at day 3,5 and 15 after operation was evaluated and recorded by the attending doctors who received professional training. The assessment methods included the National Institutes of Health Stroke Scale (NIHSS), Rey Auditory Verbal Learning Test (AVLT) and Beck Depression Inventory (BDI).

Fasting peripheral venous blood $(2 \mathrm{ml})$ was drawn from the elbow veins of patients in the two groups in the morning at day 1 before operation and at day 1,3,5 and 15 after operation using an EDTA anticoagulant tube. The blood was stored in a refrigerator at $4^{\circ} \mathrm{C}$, and the blood samples were detected within 
Table I. Comparisons of general data of patients between the two groups $(n=60)$.

\begin{tabular}{lcccr}
\hline Items & $\begin{array}{c}\text { Dexmedetomidine } \\
\text { group }\end{array}$ & $\begin{array}{c}\text { Propofol + remifentanil } \\
\text { group }\end{array}$ & $\mathrm{t} / \chi^{2}$ & P-value \\
\hline Age (years) & $61.82 \pm 6.53$ & $62.42 \pm 6.23$ & 0.5174 & 0.6059 \\
Weight (kg) & $74.33 \pm 14.36$ & $73.52 \pm 13.64$ & 0.3140 & 0.7541 \\
Sex ratio (male/female) & $34 / 26$ & $32 / 28$ & 0.1347 & 0.7136 \\
Body mass index (kg/m ${ }^{2}$ ) & $25.74 \pm 4.12$ & $26.42 \pm 4.21$ & 0.9238 & 0.3575 \\
Anesthesia time (min) & $225.56 \pm 33.45$ & $231.47 \pm 35.33$ & 0.9404 & 0.3489 \\
Operation time (min) & $187.53 \pm 27.44$ & $190.62 \pm 29.46$ & 0.5975 & 0.5513 \\
Length of stay in intensive care & $2.17 \pm 0.49$ & $2.21 \pm 0.58$ & 0.4081 & 0.6840 \\
unit (ICU) (days) & & & & \\
Length of stay in hospital (days) & $9.79 \pm 1.46$ & $9.42 \pm 1.21$ & 1.5114 & 0.1334 \\
\hline
\end{tabular}

$2 \mathrm{~h}$ after collection, while long-term standing was avoided. Peripheral venous blood $(80 \mathrm{mg})$ was taken and centrifuged at $8,000 \mathrm{x}$ g for $10 \mathrm{~min}$ to separate and purify the white blood cells. Then white blood cells were repeatedly washed with phosphate-buffered saline (PBS), purified and placed in a refrigerator at $4^{\circ} \mathrm{C}$ for detection. The mitochondrial membrane potential was detected using a BD FACSCalibur flow cytometer after staining and labeling for mitochondria in leukocytes via JC-1 fluorescence staining using the fluorescence probe MitoTracker RED CMXRose and MitoTracker Green FM at day 1 before operation and at day 1,3,5 and 15 after operation. The detection of mitochondrial membrane potential using the flow cytometer obtained a flow chart reflecting the changes in JC-1 fluorescence staining intensity. The polymer formed by JC-1 in mitochondria mainly showed the red fluorescence staining in flow cytometry. When the abnormal depolarization of mitochondrial membrane potential occurred, the mitochondria mainly showed green fluorescence staining. The lymphocytes were further separated from the purified leukocytes, followed by flow cytometry and drawing of flow chart again. The mitochondrial membrane potential in peripheral blood lymphocytes was presented through a comparison of fluorescence intensity between red and green-fluorescent mitochondria. Finally, the proportion of cells with a decline in mitochondrial membrane potential was calculated.

The absorbance of mitochondrial respiratory chain complex activity in leukocytes that were separated and purified using the above-mentioned method at day 1 before and after operation in propofol + remifentanil and dexmedetomidine groups was detected via enzyme-linked immunosorbent assay (ELISA), and it was compared with the standard curve. The concentrations of human mitochondrial respiratory chain complex I, II, III and IV in leukocytes in the dexmedetomidine group were calculated and compared with those in the propofol + remifentanil group to analyze mitochondrial function in the dexmedetomidine group.

Statistical analysis. Data in this study were processed using Statistical Product and Service Solutions (SPSS) 20.0 software (IBM, Corp., Armonk, NY, USA). Measurement data were presented as mean \pm standard deviation (SD). One-way analysis of variance or Student's t-test was used for inter- group comparison, and least significant difference (LSD) test was used for pairwise comparisons. Enumeration data were presented as a percentage (\%) and the Chi-square test was used for intergroup comparison. $\mathrm{P}<0.05$ indicated that the difference was statistically significant.

\section{Results}

General conditions of patients. A total of 120 patients undergoing elective surgery under general anesthesia were enrolled into the present study, including 54 females and 66 males, with an average age of $(62.1 \pm 6.9)$ years. There were no statistically significant differences in the comparisons of general conditions (age, body weight, sex ratio, body mass index, anesthesia time, operation time and length of stay in the ICU and hospital) between the dexmedetomidine and propofol + remifentanil groups $(\mathrm{P}>0.05)$ (Table I).

Changes in cognitive functions of patients before and after operation. There were no statistically significant differences in the NIHSS, BDI and AVLT scores for the dexmedetomidine and propofol + remifentanil groups before operation $(\mathrm{P}>0.05)$. At day 3 and 5 after operation, the NIHSS and AVLT scores in the two groups were decreased in different degrees, but the decrease range in the dexmedetomidine group was smaller than that in the propofol + remifentanil group, and the differences were statistically significant $(\mathrm{P}<0.05)$. The NIHSS and AVLT scores in the two groups recovered at day 15 after operation, and the differences were not statistically significant $(\mathrm{P}>0.05)$. At day 3, 5 and 15 after operation, the BDI scores of the two groups were increased in different degrees, but the increase range in the dexmedetomidine group was smaller than that in the propofol + remifentanil group, and the differences were statistically significant $(\mathrm{P}<0.05)$ (Figs. 1 and 2 and Tables II-IV).

Comparisons of mitochondrial membrane potentials. There was no statistically significant difference in mitochondrial membrane potential between the dexmedetomidine and propofol + remifentanil groups before operation $(\mathrm{P}>0.05)$. At day 1,3 and 5 after operation, the mitochondrial membrane potentials of the two groups were decreased in different degrees, but the decrease range in the dexmedetomidine group 
Table II. Comparisons of NIHSS scores before and after operation (mean $\pm \mathrm{SD}, \mathrm{n}=60$ ).

\begin{tabular}{lllll}
\hline Groups & $\begin{array}{l}\text { Day 1 before } \\
\text { operation }\end{array}$ & $\begin{array}{l}\text { Day 3 after } \\
\text { operation }\end{array}$ & $\begin{array}{l}\text { Day 5 after } \\
\text { operation }\end{array}$ & $\begin{array}{l}\text { Day 15 after } \\
\text { operation }\end{array}$ \\
\hline Dexmedetomidine & $11.55 \pm 3.96$ & $8.36 \pm 1.24^{\mathrm{a}}$ & $9.57 \pm 1.25^{\mathrm{a}}$ & $11.19 \pm 1.1$ \\
Propofol + remifentanil & $11.43 \pm 4.31$ & $6.48 \pm 1.11^{\mathrm{b}}$ & $7.42 \pm 1.13^{\mathrm{b}}$ & $9.25 \pm 1.33^{\mathrm{b}}$ \\
t-test & 1.251 & 6.987 & 6.015 & 5.554 \\
P-value & 0.8741 & 0.0000 & 0.0000 & 0.0565 \\
\hline
\end{tabular}

${ }^{\mathrm{a} C o m p a r e d}$ with the dexmedetomidine group at day 1 before operation, $\mathrm{P}<0.05$; ${ }^{\mathrm{b}}$ Compared with the propofol + remifentanil group at day 1 before operation, $P<0.05$. NIHSS, National Institutes of Health Stroke Scale.

Table III. Comparisons of BDI results of patients (mean $\pm \mathrm{SD}, \mathrm{n}=60$ ).

\begin{tabular}{lcccc}
\hline Groups & Day 1 before operation & Day 3 after operation & Day 5 after operation & Day 15 after operation \\
\hline Dexmedetomidine & $4.42 \pm 1.41$ & $8.48 \pm 1.86^{\mathrm{a}}$ & $7.11 \pm 1.74^{\mathrm{a}}$ & $6.22 \pm 1.28^{\mathrm{a}}$ \\
Propofol + remifentanil & $4.33 \pm 1.01$ & $13.17 \pm 2.03^{\mathrm{b}}$ & $10.05 \pm 1.26^{\mathrm{b}}$ & $7.98 \pm 1.05^{\mathrm{b}}$ \\
t-test & 1.114 & 7.826 & 7.114 & 6.865 \\
P-value & 0.6885 & 0.0000 & 0.0000 & 0.0015 \\
\hline
\end{tabular}

${ }^{\mathrm{a} C}$ Compared with the dexmedetomidine group at day 1 before operation, $\mathrm{P}<0.05$; ${ }^{\mathrm{b} C o m p a r e d}$ with the propofol + remifentanil group at day 1 before operation, $\mathrm{P}<0.05$. BDI, Beck Depression Inventory.

Table IV. Comparisons of AVLT results of patients (mean $\pm \mathrm{SD}, \mathrm{n}=60$ ).

\begin{tabular}{lcccc}
\hline Groups & Day 1 before operation & Day 3 after operation & Day 5 after operation & Day 15 after operation \\
\hline Dexmedetomidine & $40.54 \pm 6.86$ & $35.69 \pm 3.21^{\mathrm{a}}$ & $39.24 \pm 3.07$ & $40.25 \pm 5.92$ \\
Propofol + remifentanil & $40.63 \pm 7.33$ & $29.27 \pm 2.43^{\mathrm{b}}$ & $35.28 \pm 2.13^{\mathrm{b}}$ & $39.58 \pm 6.26$ \\
t-test & 1.027 & 6.538 & 6.217 & 1.526 \\
P-value & 0.9448 & 0.0000 & 0.0005 & 0.6058
\end{tabular}

${ }^{\mathrm{a} C o m p a r e d}$ with the dexmedetomidine group at day 1 before operation, $\mathrm{P}<0.05$; ${ }^{\mathrm{b} C o m p a r e d}$ with the propofol + remifentanil group at day 1 before operation, $\mathrm{P}<0.05$. AVLT, Rey Auditory Verbal Learning Test.

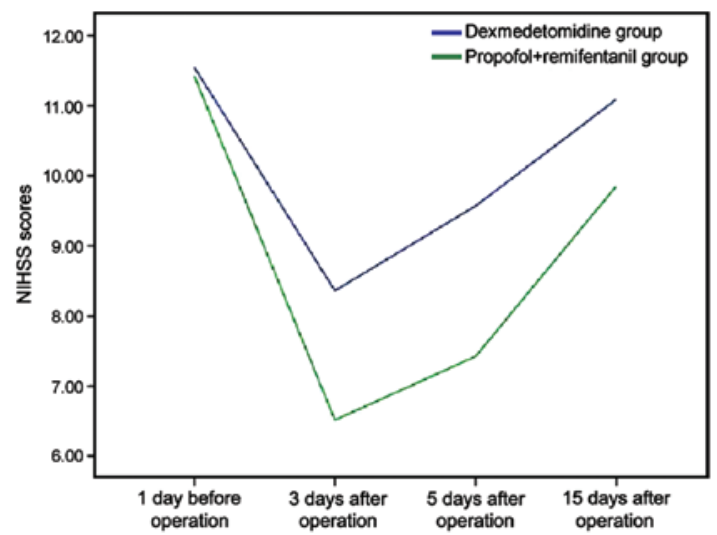

Figure 1. Line graph of comparisons of NIHSS scores before and after operation. At day 3 and 5 after operation, the NIHSS scores in the two groups are decreased, but the decrease range in the dexmedetomidine group is smaller than that in the propofol + remifentanil group, and the differences are statistically significant $(\mathrm{P}<0.05)$. The NIHSS scores in the two groups recovered at day 15 after operation, and the differences are not statistically significant $(\mathrm{P}>0.05)$. NIHSS, National Institutes of Health Stroke Scale.

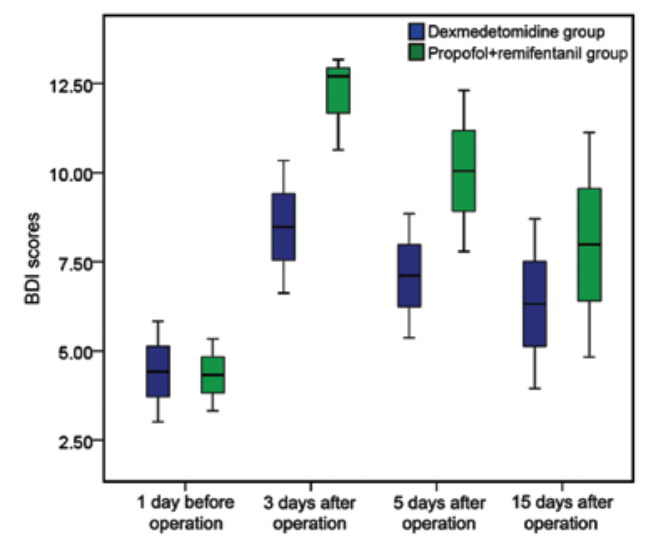

Figure 2. Box graph of comparisons of BDI results of patients. At day 3 and 5 after operation, the BDI scores in the two groups are increased, but the increase range in the dexmedetomidine group is smaller than that in the propofol + remifentanil group, and the differences are statistically significant $(\mathrm{P}<0.05)$. The BDI scores in the two groups recover at day 15 after operation, but they are significantly higher than those at day 1 before operation, and the differences are statistically significant $(\mathrm{P}<0.05)$. BDI, Beck Depression Inventory. 
Table V. Comparisons of mitochondrial membrane potentials before and after operation ( $m e a n \pm S D, n=60)$.

\begin{tabular}{lcccc}
\hline Groups & Day 1 before operation & Day 3 after operation & Day 5 after operation & Day 15 after operation \\
\hline Dexmedetomidine & $0.29 \pm 0.08$ & $0.22 \pm 0.08^{\mathrm{a}}$ & $0.24 \pm 0.07^{\mathrm{a}}$ & $0.28 \pm 0.09$ \\
Propofol + remifentanil & $0.28 \pm 0.09$ & $0.16 \pm 0.07^{\mathrm{b}}$ & $0.19 \pm 0.06^{\mathrm{b}}$ & $0.22 \pm 0.08^{\mathrm{b}}$ \\
t-test & 0.5213 & & & 0.0001 \\
P-value & 0.0000 & 0.002 \\
\hline
\end{tabular}

${ }^{\mathrm{a} C o m p a r e d}$ with the dexmedetomidine group at day 1 before operation, $\mathrm{P}<0.05$; ${ }^{\mathrm{b}}$ Compared with the propofol + remifentanil group at day 1 before operation, $\mathrm{P}<0.05$.

Table VI. Comparisons of mitochondrial respiratory chain complex activities $[\mu \mathrm{mol} /(\mathrm{min} \cdot \mathrm{mg}), \mathrm{n}=60]$.

\begin{tabular}{|c|c|c|c|c|c|c|}
\hline \multirow[b]{2}{*}{ Mitochondrial respiratory chain complexes } & \multicolumn{2}{|c|}{ Propofol + remifentanil group } & \multirow[b]{2}{*}{$\mathrm{P}$-value } & \multicolumn{2}{|c|}{ Dexmedetomidine group } & \multirow[b]{2}{*}{ P-value } \\
\hline & $\begin{array}{c}\text { Day } 1 \text { before } \\
\text { operation }\end{array}$ & $\begin{array}{c}\text { Day } 1 \text { after } \\
\text { operation }\end{array}$ & & $\begin{array}{c}\text { Day } 1 \text { before } \\
\text { operation }\end{array}$ & $\begin{array}{c}\text { Day } 1 \text { after } \\
\text { operation }\end{array}$ & \\
\hline I & $5.6 \pm 2.7$ & $4.2 \pm 2.3$ & $<0.05$ & $5.8 \pm 2.8$ & $5.5 \pm 2.5^{\mathrm{a}}$ & $>0.05$ \\
\hline II & $15.5 \pm 4.1$ & $11.5 \pm 2.8$ & $<0.05$ & $15.9 \pm 4.3$ & $14.6 \pm 3.9^{\mathrm{a}}$ & $>0.05$ \\
\hline III & $22.9 \pm 4.8$ & $17.4 \pm 4.9$ & $<0.05$ & $22.7 \pm 5.1$ & $20.4 \pm 5.7^{\mathrm{a}}$ & $>0.05$ \\
\hline IV & $145.6 \pm 19.9$ & $131.6 \pm 16.8$ & $<0.05$ & $147.9 \pm 19.8$ & $140.8 \pm 18.7^{\mathrm{a}}$ & $>0.05$ \\
\hline
\end{tabular}

${ }^{a}$ There is a statistically significant difference at day 1 after operation between dexmedetomidine and propofol + remifentanil groups $(\mathrm{P}<0.05)$.

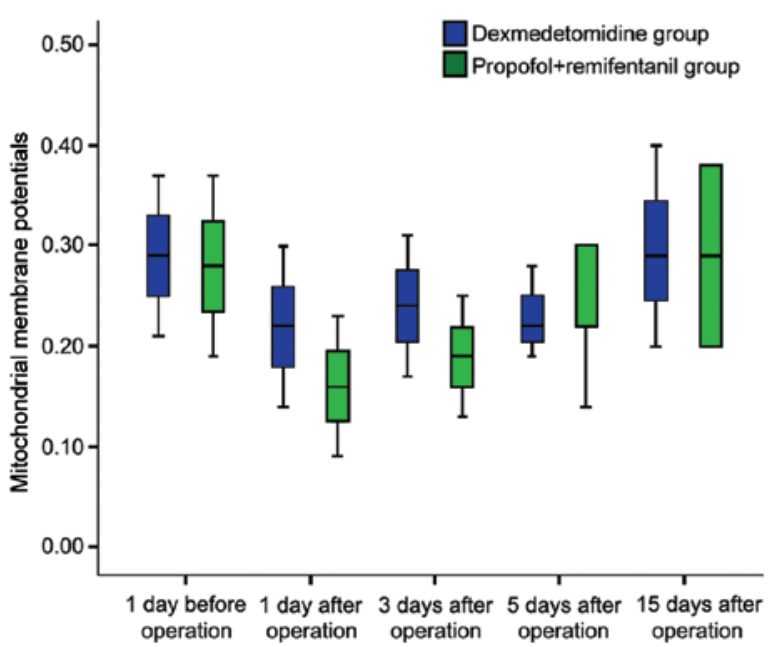

Figure 3. Box graph of comparisons of mitochondrial membrane potentials before and after operation. At day 1,3 and 5 after operation, the mitochondrial membrane potentials of the two groups are decreased, but the decrease range in the dexmedetomidine group is smaller than that in the propofol + remifentanil group, and the differences are statistically significant $(\mathrm{P}<0.05)$. In addition, the mitochondrial membrane potentials of the two groups returned to the preoperative levels at day 15 after operation, and the differences were not statistically significant $(\mathrm{P}>0.05)$.

was smaller than that in the propofol + remifentanil group, and the differences were statistically significant $(\mathrm{P}<0.05)$. The mitochondrial membrane potentials of the two groups returned to the preoperative levels at day 15 after operation, and the differences were not statistically significant $(\mathrm{P}>0.05)$ (Fig. 3 and Table V).
Comparisons of mitochondrial respiratory chain complex activities of the two groups. The activities of mitochondrial respiratory chain complexes I-IV in the propofol + remifentanil group at day 1 after operation were significantly decreased compared with those before operation, and the differences were statistically significant $(\mathrm{P}<0.05)$. The activities of mitochondrial respiratory chain complexes I-IV in the propofol + remifentanil group at day 1 after operation were decreased slightly compared with those before operation, and the differences were not statistically significant $(\mathrm{P}>0.05)$. The decrease in activities of mitochondrial respiratory chain complexes I-IV in the propofol + remifentanil group at day 1 after operation was more significant than that in the dexmedetomidine group and the difference was statistically significant $(\mathrm{P}<0.05)$, suggesting that the activities of mitochondrial respiratory chain complexes were obviously decreased in the propofol + remifentanil group (Table VI).

\section{Discussion}

Cognitive function is the advanced neurophysiological function of the human brain. POCD occurs frequently in elderly patients receiving operation under general anesthesia, and it can be divided into early POCD (within 7 days after operation) and long-term POCD ( $>3$ months after operation) according to the POCD duration (7). Manifestations of POCD patients include amnesia and reactive disorder to instructions, and severe patients are accompanied with personality changes (7-9). POCD can occur on the day after general anesthesia or after several months to years, and the majority of patients has a shorter duration of disease and can recover spontaneously 
after operation. Long-term POCD may seriously affect the quality of life of patients (7). POCD is an external manifestation of central nervous system injury. The incidence of POCD in patients undergoing cardiopulmonary bypass is as high as $10-50 \%$, while the incidence of POCD is $35 \%$ in adults after coronary artery bypass grafting, and the disease is particularly severe and enduring in elderly patients with POCD. Studies have shown that dexmedetomidine can exert its neuroprotective effect by alleviating neuronal damage in the hippocampal CA1 region of rat model of cerebral ischemia. In neonatal rats, dexmedetomidine can reduce the isoflurane-induced hippocampal, thalamic and cortical damage to improve the postoperative neurological cognitive function. Moreover, the combined administration of dexmedetomidine and isoflurane may reduce the neurotoxicity of isoflurane $(9,10)$.

Strong stress response caused by general anesthesia and surgery can produce a large number of oxygen free radicals in the human body, and anesthetics can affect the mitochondrial function, free radical production and neuronal apoptosis during perioperative period. Inhalation of anesthetics, such as sevoflurane and isoflurane, can improve mitochondrial function and energy metabolism and inhibit the release of free radicals, thus protecting the ischemia reperfusion injury of brain, liver and other organs. Oxidative stress-induced neuronal mitochondrial membrane electrophysiological dysfunction is a core step of oxidative stress-induced neuronal apoptosis (10-12). JC-1 is an ideal fluorescence probe widely used to detect the mitochondrial membrane potential. In addition, JC-1 dyes accumulate in mitochondria in a potential-dependent manner, which can be used to detect cell, tissue or purified mitochondrial membrane potentials. In this study, the neuronal mitochondrial membrane potentials of patients before and after operation were measured, and the results showed that the decline in neuronal mitochondrial membrane potential of patients receiving dexmedetomidine during surgery was smaller and the recovery rate was higher with time, indicating that the production of oxygen-free radicals is accelerated after the strong stress of general anesthesia and operation, the ultra-high oxidative stress level allows the permeability transition pore of neuronal mitochondrial membrane to remain continuously open (13-15), and dexmedetomidine can alleviate this process.

Under the physiological state, the cells in the body can generate free radicals and other oxidants, but the generation/elimination rate is in a balanced state without causing oxidative stress injury. However, the general anesthesiainduced neuronal damage leads to the formation of reactive oxygen and nitrogen species (such as superoxide radical anions, hydroxyl radicals, nitric oxide and peroxynitrite), all of which are involved in neuronal damage. In the case of cerebral ischemia, the brain tissues are subjected to anoxia or hypoxia due to the obstruction of arterial blood supply or insufficient blood flow $(12,13)$. Ischemia-reperfusion injury of brain tissues causes ischemia and hypoxia for neuronal cells, resulting in increased catabolism of ATP, and a large amount of its metabolite hypoxanthine accumulates in neurons (16). The aggravated oxidative stress uses up the endogenous antioxidant in the body, leading to neurons producing a large number of oxygen free radicals (17-19). Neuronal mitochondrion is another important source of oxygen-free radicals, and neuronal ischemia-reperfusion injury can decrease the mitochondrial membrane potential, thus leading to mitochondrial respiratory chain dysfunction (20-22). The present study showed that the activities of mitochondrial respiratory chain complexes I-IV in the propofol + remifentanil group at day 1 after operation were significantly decreased compared with those before operation $(\mathrm{P}<0.05)$. By contrast, activities in the dexmedetomidine group were only slightly decreased compared with those before operation $(\mathrm{P}>0.05)$. The decline in activities of mitochondrial respiratory chain complexes I-IV at day 1 after operation in the propofol + remifentanil group was more significant than that in the dexmedetomidine group $(\mathrm{P}<0.05)$, suggesting that the application of dexmedetomidine alleviates the reduction in mitochondrial respiratory chain complex activity to a certain extent.

In conclusion, dexmedetomidine can relieve the neuronal damage that may be caused by mitochondrial membrane oxidative stress, alleviate the damage to mitochondrial-related enzyme system activity and reduce the damage to the activities of mitochondrial respiratory chain enzyme complexes I, II, III and IV, ultimately improving the POCD of patients.

\section{References}

1. Cascella M, Muzio MR, Bimonte S, Cuomo A and Jakobsson JG: Postoperative delirium and postoperative cognitive dysfunction: Updates in pathophysiology, potential translational approaches to clinical practice and further research perspectives. Minerva Anestesiol: Oct 4, 2017 (Epub ahead of print).

2. Alam A, Suen KC, Hana Z, Sanders RD, Maze M and Ma D: Neuroprotection and neurotoxicity in the developing brain: An update on the effects of dexmedetomidine and xenon. Neurotoxicol Teratol 60: 102-116, 2017.

3. Sun X, Xu H, Shen J, Guo S, Shi S, Dan J, Tian F, Tian Y and Tian Y: Real-time detection of intracellular reactive oxygen species and mitochondrial membrane potential in THP-1 macrophages during ultrasonic irradiation for optimal sonodynamic therapy. Ultrason Sonochem 22: 7-14, 2015.

4. Bai X, Song Z, Zhou Y, Pan S, Wang F, Guo Z, Jiang M, Wang G, Kong R and Sun B: The apoptosis of peripheral blood lymphocytes promoted by hyperbaric oxygen treatment contributes to attenuate the severity of early stage acute pancreatitis in rats. Apoptosis 19: 58-75, 2014.

5. Ning Q, Liu Z, Wang X, Zhang R, Zhang J, Yang M, Sun H, Han F, Zhao W and Zhang X: Neurodegenerative changes and neuroapoptosis induced by systemic lipopolysaccharide administration are reversed by dexmedetomidine treatment in mice. Neurol Res 39: 357-366, 2017.

6. Liu Y, Ma L, Gao M, Guo W and Ma Y: Dexmedetomidine reduces postoperative delirium after joint replacement in elderly patients with mild cognitive impairment. Aging Clin Exp Res 28: 729-736, 2016.

7. Hovens IB, Schoemaker RG, van der Zee EA, Absalom AR, Heineman E and van Leeuwen BL: Postoperative cognitive dysfunction: Involvement of neuroinflammation and neuronal functioning. Brain Behav Immun 38: 202-210, 2014.

8. Montagne A, Barnes SR, Sweeney MD, Halliday MR, Sagare AP, Zhao Z, Toga AW, Jacobs RE, Liu CY, Amezcua L, et al: Blood-brain barrier breakdown in the aging human hippocampus. Neuron 85: 296-302, 2015.

9. Xu J, Hu H, Chen B, Yue R, Zhou Z, Liu Y, Zhang S, Xu L, Wang $\mathrm{H}$ and $\mathrm{Yu} \mathrm{Z}$ : Lycopene protects against hypoxia/reoxygenation injury by alleviating ER stress induced apoptosis in neonatal mouse cardiomyocytes. PLoS One 10: e0136443, 2015.

10. Wang Y, Han R and Zuo Z: Dexmedetomidine-induced neuroprotection: Is it translational? Transl Perioper Pain Med 1: 15-19, 2016.

11. Wu HJ, Wu HB, Zhao YQ, Chen LJ and Zou HZ: Bergamottin isolated from Citrus bergamia exerts in vitro and in vivo antitumor activity in lung adenocarcinoma through the induction of apoptosis, cell cycle arrest, mitochondrial membrane potential loss and inhibition of cell migration and invasion. Oncol Rep 36: 324-332, 2016. 
12. Pan H, Xie X, Chen D, Zhang J, Zhou Y and Yang G: Protective and biogenesis effects of sodium hydrosulfide on brain mitochondria after cardiac arrest and resuscitation. Eur J Pharmacol 741: 74-82, 2014.

13. Thompson JW, Narayanan SV, Koronowski KB, Morris-Blanco K, Dave KR and Perez-Pinzon MA: Signaling pathways leading to ischemic mitochondrial neuroprotection. J Bioenerg Biomembr 47: 101-110, 2015.

14. Liu XZ, Wang FC, Wang Y and Zhang JX: Efficacy of a combination of taurine and stenosis removing on cognitive impairment induced by carotid artery stenosis in rats. Eur Rev Med Pharmacol Sci 21: 1884-1890, 2017.

15. Yang L, Zhu L, Dong W, Cao Y, Lin L, Rong Z, Zhang Z and Wu G: Reactive oxygen species-mediated mitochondrial dysfunction plays a critical role in high glucose-induced nucleus pulposus cell injury. Int Orthop 38: 205-206, 2014.

16. Bellanti F, Mirabella L, Mitarotonda D, Blonda M, Tamborra R, Cinnella G, Fersini A, Ambrosi A, Dambrosio M, Vendemiale G, et al: Propofol but not sevoflurane prevents mitochondrial dysfunction and oxidative stress by limiting HIF-1 $\alpha$ activation in hepatic ischemia/reperfusion injury. Free Radic Biol Med 96: 323-333, 2016.

17. Stetler RA, Leak RK, Yin W, Zhang L, Wang S, Gao Y and Chen J: Mitochondrial biogenesis contributes to ischemic neuroprotection afforded by LPS pre-conditioning. J Neurochem 123 (Suppl 2): 125-137, 2012.
18. Sharma J, Johnston MV and Hossain MA: Sex differences in mitochondrial biogenesis determine neuronal death and survival in response to oxygen glucose deprivation and reoxygenation. BMC Neurosci 15: 9, 2014.

19. Akman T, Guven M, Aras AB, Ozkan A, Sen HM, Okuyucu A, Kalkan Y, Sehitoglu I, Silan C and Cosar M: The neuroprotective effect of glycyrrhizic acid on an experimental model of focal cerebral ischemia in rats. Inflammation 38: 1581-1588, 2015.

20. Qian XL, Zhang W, Liu MZ, Zhou YB, Zhang JM, Han L, Peng YM, Jiang JH and Wang QD: Dexmedetomidine improves early postoperative cognitive dysfunction in aged mice. Eur J Pharmacol 746: 206-212, 2015.

21. Chouchani ET, Pell VR, James AM, Work LM, Saeb-Parsy K, Frezza C, Krieg T and Murphy MP: A unifying mechanism for mitochondrial superoxide production during ischemia-reperfusion injury. Cell Metab 23: 254-263, 2016.

22. Sinha K, Das J, Pal PB and Sil PC: Oxidative stress: The mitochondria-dependent and mitochondria-independent pathways of apoptosis. Arch Toxicol 87: 1157-1180, 2013.

This work is licensed under a Creative Commons Attribution-NonCommercial-NoDerivatives 4.0 International (CC BY-NC-ND 4.0) License. 